\title{
DOOM'd to switch: superior cognitive flexibility in players of first person shooter games
}

\author{
Lorenza S. Colzato ${ }^{*}$, Pieter J.A. van Leeuwen ${ }^{1}$, Wery P. M. van den Wildenberg ${ }^{2}$ and Bernhard Hommel' \\ Cognitive Psychology Unit and Leiden Institute for Brain and Cognition, Leiden University, Leiden, Netherlands \\ Amsterdam Center for the Study of Adaptive Control in Brain and Behavior (Acacia), Department of Psychology, University of Amsterdam, Amsterdam, Netherlands
}

Edited by:

Wilfried Kunde, Dortmund University,

Germany

Reviewed by:

Andrea Kiesel, Julius-Maximilians-

University, Germany

*Correspondence:

Lorenza S. Colzato, Leiden University,

Department of Psychology, Cognitive

Psychology Unit, Postbus 9555, 2300

RB Leiden, Netherlands.

e-mail:colzato@fsw.leidenuniv.nl
The interest in the influence of videogame experience on our daily life is constantly growing. "First Person Shooter" (FPS) games require players to develop a flexible mindset to rapidly react to fast moving visual and auditory stimuli, and to switch back and forth between different subtasks. This study investigated whether and to which degree experience with such videogames generalizes to other cognitive-control tasks. Video-game players (VGPs) and individuals with little to no videogame experience (NVGPs) performed on a task switching paradigm that provides a relatively well-established diagnostic measure of cognitive flexibility. As predicted, VGPs showed smaller switching costs (i.e., greater cognitive flexibility) than NVGPs. Our findings support the idea that playing FPS games promotes cognitive flexibility.

Keywords: videogame, task-switching, cognitive flexibility

\section{INTRODUCTION}

In the last decade the gaming industry has seen a rapid growth, as did the number of their customers. Exciting new technologies are available and the variety of games available is enormous, so that players can be found at any age and socio-economical background. This has raised the interest of scientists, who often focus on the influence of videogame experience on our daily behavior, especially with regard to particularly violent games. Politicians and main-stream media are mostly concerned about (possible, but not yet scientifically confirmed) extreme social and behavioral effects, such as violence or addiction, guided by the famous tragic cases in high-school amok run teenagers in the USA and, recently, also in Germany, who were used to play "First Person Shooter" games.

Compared to the amount of attention these possible negative effects receive, very little research has been done on possible positive effects that videogame experience might have on cognitive skills and abilities. Recent studies on the differences in cognitive skills between video-game players (VGPs) and non-video-game players (NVGPs) have shown surprising and theoretically interesting effects on various perceptual tasks. Trick et al. (2005) found VGPs to outperform NVGPs in visually tracking multiple moving objects across a display crowded with distracters. VGPs also showed better performance in both easy and difficult visual search tasks (Castel et al., 2005). Green and Bavelier $(2003,2006 a, b, 2007)$ demonstrated that videogame experience modulates different aspects of visual selective attention. A flanker compatibility task was used to measure an increase in attentional capacity and an adapted "useful field of view" task assessed the critical areas of the visual field. The results showed that VGPs performed better than NVGPs across the whole field of view. Importantly, attention was also affected in NVGPs that were trained for several months on action videogames, confirming the causal role of gaming experience.

The new generation of "First Person Shooter" (FPS) games (compared to older generation games) are not just about pressing one button at the right moment, but require the players to develop a flexible mindset that allows them to engage in complex scenarios, to rapidly react to moving visual and sudden acoustic events, and to switch back and forth between different subtasks. The goal of the present study was to test the hypothesis that gaming is associated with a general and generalizable enhancement of cognitive flexibility. If so, more extensive experience with videogames should be associated with better performance on a task that requires mental flexibility, such as task switching (Miyake et al., 2000; Monsell, 2003). Switching from one task to another requires the reconfiguration of the cognitive task set, a process that has often been found to delay reaction time on the first post-switch trial - the so-called task-switching costs (Allport et al., 1994). If playing FPS games would indeed be associated with higher flexibility, VGPs should show smaller task-switching costs than NVGPs.

\section{MATERIALS AND METHODS PARTICIPANTS}

Table 1 presents the participant characteristics. Thirty-four young healthy adults served as participants, consisting of one group of 17 video-game players (VGPs) and 17 individuals with little to no videogame experience (NVGPs). Participants were recruited among students and through advertisements on internet forums mainly visited by VGPs. VGPs met the following criteria: play video games at least four times a week for a minimum period of 6 months. Subjects playing mainly web-based puzzle games were excluded. VGPs played on multiple platforms (PC, Xbox, Playstation and Nintendo). VGPs participating in the study played mainly FPS games, such as Call of Duty: Modern Warfare, Unreal Tournament, Battlefield, and the very controversial Grand Theft Auto IV. All of these games are situated in a 3D environment and require frequent switching between multiple tasks.

\section{APPARATUS AND STIMULI}

The experiment was controlled by a Windows-operated computer attached to a Philips $17^{\prime \prime}$ monitor. Responses were made by pressing the "Z" or "?" of the QWERTY computer keyboard with the left and 
Table 1 | Demographic characteristics, mean response latencies (in $\mathrm{ms}$ ), error rates (in percent), and switching costs (alternation - repetition) for VGPs and NVGPs.

\begin{tabular}{lll}
\hline Variables (SE) & VGPs & NVGPs \\
\hline Age & $24(3.5)$ & $23(4.5)$ \\
IQ & $105(6.5)$ & $107(4.8)$ \\
REPETITION & & \\
Reaction times (ms) & $415(21)$ & $439(21)$ \\
Error rates (\%) & $4.3(0.9)$ & $4.2(0.9)$ \\
ALTERNATION & & $535(27)$ \\
Reaction times (ms) & $459(27)$ & $2.6(0.9)$ \\
Error rates (\%) & $5.2(0.9)$ & \\
SWITCH COSTS & & $96^{*}$ \\
Reaction times (ms) & $44^{*}$ & -1.6 \\
Error rates (\%) & 0.9 & \\
\hline
\end{tabular}

Standard errors are presented in parentheses.

Ns: Non-significant difference.

Significant group difference; ${ }^{*} p<0.05,{ }^{* *} p<0.01$.

right index finger, respectively. The target stimuli were adopted from Huizinga et al. (2006), and consisted of geometric figures. Larger (global) rectangles/squares consisted of smaller (local) rectangles or squares. Global stimuli (i.e., squares or rectangles; $93 \times 93$ pixels or $41 \times 189$ pixels, respectively) were composed of many smaller "local" stimuli (i.e., squares or rectangles; $21 \times 21$ pixels or $8 \times 46$ pixels, respectively). The space between the local elements of a stimulus was three pixels. A global square consisted of 16 small squares or 16 small rectangles; a global rectangle consisted of 16 small squares or 16 small rectangles. The "local" and "global" cues were the same size as the global and local stimuli and were presented at 189 pixels from the center of the computer screen.

\section{PROCEDURE AND DESIGN}

All participants were tested individually and completed a fluidintelligence test and the switching task. Individual IQ was determined by means of a 30-min reasoning-based intelligence test (Raven's Standard Progressive Matrices: SPM; Raven et al., 1988). The SPM assesses the individual's ability to create perceptual relations and to reason by analogy independent of language and formal schooling; it is a standard, widely used test to measure Spearman's $\mathrm{g}$ factor and of fluid intelligence in particular.

Participants responded to randomly presented rectangles or squares by pressing a left or right response button, respectively. Three blocks of trials were administered, two training blocks in which the instruction (global or local) was constant across all trials, followed by the experimental block in which participants switched between the global and the local task. In one of the two training blocks, participants responded to the local figure, in the other block they responded to the global figure. The order of the training blocks was randomized across participants and each block consisted of 50 trials. In the third block consisting of 160 trials, participants alternated between predictable sequences of four "local" and four "global" trials). A cue indicated to which dimension (global or local) the participants should respond. Cues that related to the global (local) dimension consisted of a big (small) square, presented at one side of the target stimulus, and a big (small) rectangle, presented at the other side of the target stimulus (see Figure 1). The color of cues and target was red. Both remained on the screen until a response was given or $2500 \mathrm{~ms}$ had passed. The time interval between presentation of the cue and of the target stimulus varied between 400 and $500 \mathrm{~ms}$ and the interval between responses and the next presentation of the cue varied between 900 and $1100 \mathrm{~ms} \mathrm{~ms}$.

\section{STATISTICAL ANALYSIS}

Independent samples t-tests were performed to test Age and IQ values between groups. Mean RTs and proportions of errors were analyzed by means of ANOVAs using target level (global vs. local), the congruency between the stimuli on the two levels (congruent vs. incongruent), and task switch (i.e., same vs. different target level as in previous trial: task repetition vs. alternation) as withinand Group (VGPs vs. NVGPs) as between-participants factor. A significance level of $\mathrm{p}<0.05$ was adopted for all tests.

\section{RESULTS}

No significant differences between the two groups were found for age $t(32)=1.47, p=0.15$ and $\mathrm{IQ}, t(32)=0.57, p=0.57$.

The reaction time analysis showed no evidence of a group effect, $F(1,32)=2.47, p=0.13, \mathrm{MSE}=71441.53, \eta^{2} p=0.07$, but the remaining three main effects were reliable (see Table 2). First, the effect of switch, $F(1,32)=35.48, p<0.0001$, MSE $=9488.07$, $\eta^{2} p=0.53$, was due to that repeating the task allowed for faster responding than switching between target levels (427 vs. $497 \mathrm{~ms}$ ). Second, the effect of target level, $F(1,32)=65.58, p<0.0001$, $\mathrm{MSE}=2447.90, \eta^{2} p=0.67$, reflected the well-known global preference (Navon, 1977), that is, faster responses to globally than locally defined targets (438 vs. $486 \mathrm{~ms}$ ). Third, the congruency effect, $F(1,32)=23.74, p<0.0001, \mathrm{MSE}=2570.35, \eta^{2} p=0.43$, indicated interference from the non-target level, that is, faster responses if the stimulus at the currently irrelevant level was congruent with the present target than if that stimulus was incongruent (447 vs. $477 \mathrm{~ms})$.

More important for present purposes, the size of the switch effect varied with group, $F(1,32)=4.92, p<0.05$, $\mathrm{MSE}=9488.07$, $\eta^{2} p=0.13$. As suggested by Figure 2 , switching costs were reliable for both VGPs and NVGPs, $F(1,16)=14.49, p<0.001$, $\mathrm{MSE}=4574.42, \eta^{2} p=0.47$ and $F(1,16)=22.01, p<0.0001$, MSE $=14401.71, \eta^{2} p=0.58$, respectively (see Table 1). However, as predicted, switch costs were reduced in VGPs. This reduction was due to better performance on switch trials, suggesting that videogame experience selectively targeted the condition in which cognitive control was needed most. No other interaction was reliable.

The analysis of the error rates revealed two reliable main effects. First, the effect of congruency, $F(1,32)=38.51, p<0.0001$, $\mathrm{MSE}=44.51, \eta^{2} p=0.55$, reflecting the interference of the irrelevant target level, as indicated by a smaller proportion of errors on congruent as compared to incongruent trials ( $1.5 \%$ vs. $6.7 \%)$. Second, the effect of target level, $F(1,32)=7.08, p<0.05, \mathrm{MSE}=40.19$, $\eta^{2} p=0.18$, suggesting less errors to globally than locally defined targets $(3.1 \%$ vs. $5.1 \%)$. No other effect was significant. 


\section{DISCUSSION}

Our findings show that videogame experience is associated with cognitive flexibility as measured by a task-switching paradigm: VGPs showed smaller switching costs than NVGPs, suggesting that they have better cognitive-control skills. As no significant group differences were found for age or estimated IQ, we can rule out an account of our results in these terms - which is an important conclusion given the evidence that cognitive flexibility varies with age (Kray et al., 2002) and fluid intelligence (Colzato et al. 2006).

The observation that playing FPS games predicts performance on a relatively well-established diagnostic index of cognitive flexibility (Miyake et al., 2000; Monsell, 2003) may provide some support for

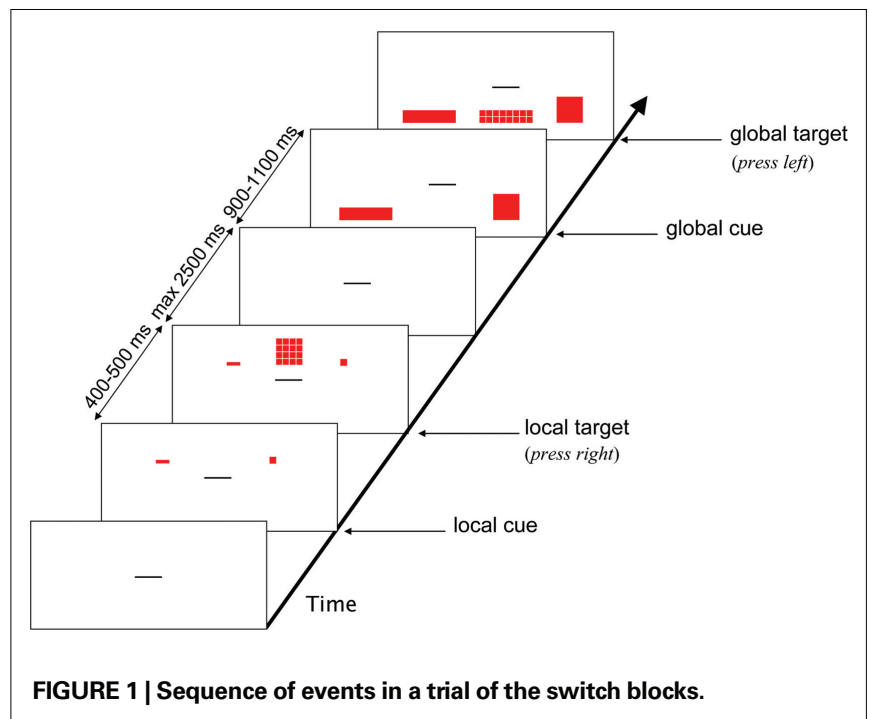

the idea that videogame experience enhances cognitive flexibility in general. Switching costs in tasks as used in the present study are thought to consist of several components (e.g., Meiran et al., 2000). One of the two major components (the preparatory component) is reduced as the preparation interval increases and often eliminated at long intervals (Meiran, 1996). The other major component is considered to be "residual", that is, resistant to preparation. Even though there is no agreement in the field how to interpret these residual costs (e.g., Altmann, 2003; Monsell, 2003), there is rather strong evidence that they result from the involuntary, presumably stimulus-triggered activation of the previous task set. For instance, the size of residual switching costs is dramatically reduced if the present stimulus is novel and has not yet been presented in the context of a competing task (Waszak et al., 2003). Given that the cuetarget interval used in the present study was rather long, we assume that video game experience affects, or is associated with, residual switch costs - while our design was not suited to assess possible effects on the preparatory component. We speculate that VGPs are more efficient in controlling episodic memory structures and, thus, in selectively activating and updating task sets. This idea would fit with the recent demonstration that neurofeedback that enhances neural synchronization in the frontal cortex leads to a more selective retrieval from episodic memory, including the suppression of automatically retrieved but interfering episodic memory traces (Keizer et al., 2010a,b).

An alternative explanation of our results may be that VGPs, who are often accused of being antisocial or aggressive in the media, might have been more intrinsically motivated than NVGPs to accomplish the task. However, the data do not provide strong support for this possibility: even though VGPs were somewhat (but not significantly) faster than NVGPs, they tended to be somewhat (but not significantly) less accurate, suggesting that the overall performance in the two groups was rather comparable.

Table 2 | Means of mean reaction times for responses (RT; in $\mathrm{ms}$ ) and percentages of errors (PE), as a function of group (VGPs vs. VGPs), task switch (i.e., same vs. different target level as in previous trial: task repetition vs. alternation), target level (global vs. local) and the congruency between the stimuli on the two levels (congruent vs. incongruent).

Variables (SE)

VGPs

Switch

\begin{tabular}{|c|c|c|c|c|c|c|c|c|}
\hline \multirow{2}{*}{$\begin{array}{l}\text { Target } \\
\text { Congruency }\end{array}$} & \multicolumn{2}{|c|}{ Local } & \multicolumn{2}{|c|}{ Global } & \multicolumn{2}{|c|}{ Local } & \multicolumn{2}{|c|}{ Global } \\
\hline & Inc & Con & Inc & Con & Inc & Con & Inc & Con \\
\hline PE (\%) & $9.3(2.3)$ & $0.9(0.7)$ & $4.7(1.2)$ & $2.1(0.7)$ & $11.1(2.8)$ & $0.98(0.9)$ & $7.4(1.6)$ & $1.6(0.7)$ \\
\hline Variables (SE) & & & & & & & & \\
\hline
\end{tabular}

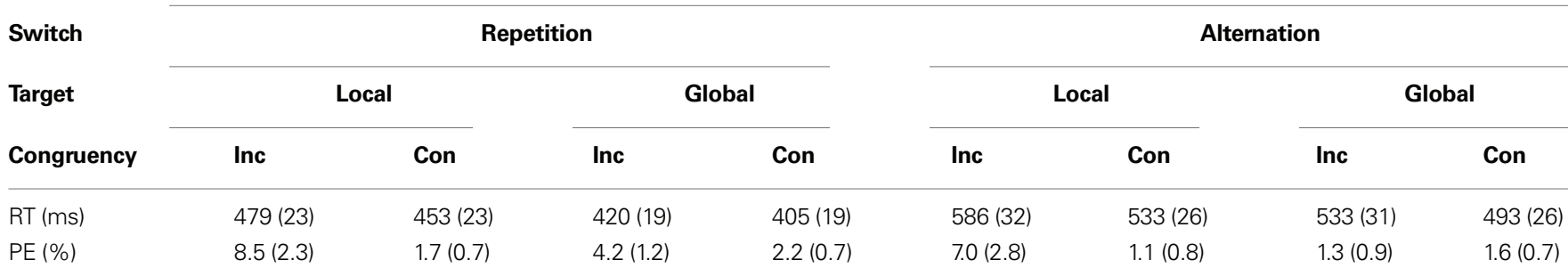




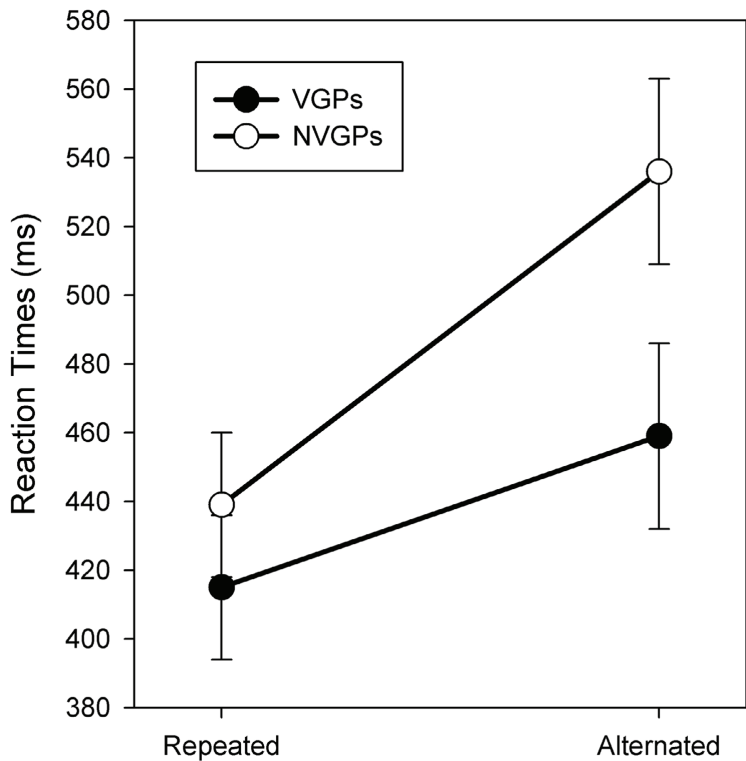

Task

FIGURE 2 | Mean reaction times (ms) as a function of group (video-game players [VGPs] vs. individuals with little to no videogame experience [NVGPs]) and task switch (i.e., same vs. different target level as in previous trial: task repetition vs. alternation). Standard errors of the difference between Task Repetition and Alternation trials are represented by the error bars.

On the one hand, our observations fit with previous reports of beneficial effects of videogame experience on cognitive skills and abilities, such as needed for visual search (Castel et al., 2005) and other visual tasks (Green and Bavelier, 2007). On the other hand, however, it is interesting to note that we did not find any impact of videogame experience on visual attention, as indicated by the absence of interactions between group and the global precedence effect, and between group and congruency. This might suggest a reinterpretation of previous findings: rather than low-level perceptual or vision-related attentional

\section{REFERENCES}

Allport, D. A., Styles, E. A., and Hsieh, S. (1994). "Shifting intentional set: Exploring the dynamic control of tasks," in Attention and Performance XV: Conscious and Nonconscious Information Processing, eds C. Umiltà and M. Moscovitch (Cambridge, MA: MIT Press, Bradford Books), 421-452.

Altmann, E. M. (2003). Task switching and the pied homunculus: where are we being led? Trends Cogn. Sci. (Regul. Ed.), 7, 340-341.

Castel, A. D., Pratt, J., and Drummond, E. (2005). The effects of action video game experience on the time course of inhibition of return and the efficiency of visual search. Acta Psychol. (Amst), 119, 217-230.
Colzato, L. S., van Wouwe, N. C., Lavender, T., and Hommel, B. (2006). Intelligence and cognitive flexibility: Fluid intelligence correlates with feature "unbinding" across perception and action. Psychon. Bull. Rev., 13, 1043-1048.

Green,C. S., and Bavelier, D. (2003).Action video game modifies visual selective attention. Nature 423, 534-537.

Green, C. S., and Bavelier, D. (2006a). Enumeration versus multiple object tracking: the case of action video game players. Cognition 101, 217-245.

Green, C. S., and Bavelier, D. (2006b). Effect of action video games on the spatial distribution of visuospatial attention. J. Exp. Psychol. Hum. Percept. Perform., 32, 1465-1468.

processes per se, it might be executive control functions that are improved by videogame practice. If so, experimental tasks may reflect the beneficial effects of video gaming (only) to the degree that they draw on control processes and central resources (e.g., perceptual decision-making and attentional control). Moreover, it would be interesting to look whether this sort of training effect (Minear and Shah, 2008) is specific to FPS games or whether it could also be found in dynamic 3D games (as for example Super Mario Galaxy 2) without aggressive content. From preliminary results we are lead to believe that it is in particular the first person perspective (as in the FPS games) that allows for cognitive improvement, but more systematic research on this issue is necessary.

It is important to note that the causal relation between our observations and videogame experience may not be straightforward. Indeed, we cannot exclude that preexisting neuro-developmental factors may play a mediating role. For instance, individuals with a genetic predisposition that favors executive control functions might be drawn to video games more strongly, so that what looks like an effect of practice might actually represent a kind of self-selection. However, several studies in which NVGPs were trained for several months on action videogame have shown strongly improved performance in tasks requiring good spatial resolution in vision and the efficient distribution of visual attention (Green and Bavelier, 2003, 2006a,b, 2007).

It seems of societal relevance to devote more research on the functional significance of videogame experience for the adaptive control of behaviour, for example in healthy aging. Indeed, training elderly with videogames may be a successful strategy to compensate for losses in their ability to adapt and restructure the cognitive system in response to changing situational demands $-\mathrm{a}$ skill that is essential for almost all "functional” everyday behaviour. The present findings also raise the question whether VGPs show enhancements in other cognitive-control functions as well, such as the inhibition of overt manual responses and the "updating" (and monitoring of) working memory (WM) representations the remaining two of the three major control functions (Miyake et al., 2000).

Green, C. S., and Bavelier, D. (2007) Action-video-game experience alters the spatial resolution of attention. Psychol. Sci., 18, 88-94.

Huizinga, M., Dolan, C. V., and Van der Molen, M. W. (2006). Agerelated change in executive function: Developmental trends and a latent variable analysis. Neuropsychologia 44, 2017-2036.

Keizer, A. W., Verment, R., and Hommel, B. (2010a). Enhancing cognitive control through neurofeedback: a role of gamma-band activity in managing episodic retrieval. Neuroimage 49, 3404-3413.

Keizer, A. W., Verschoor, M., Verment, R. and Hommel, B. (2010b). The effect of gamma enhancing neurofeedback on measures of feature-binding flexibility and intelligence. Int. J. Psychophysiol. 75, 25-32.

Kray, J., Li, K. Z. H., and Lindenberger, U. (2002). Age-related changes in task-switching components: The role of task uncertainty. Brain Cogn., 49, 363-381.

Meiran, N. (1996). Reconfiguration of processing mode prior to task performance. J. Exp. Psychol. Learn. Mem. Cogn., 22, 1423-1442.

Meiran, N., Chorev, Z., and Sapir, A. (2000). Component processes in task switching. Cogn. Psychol., 41, 211-253.

Minear, M., and Shah, P. (2008). Training and transfer effects in task switching. Mem. Cogn., 36, 1470-1483.

Miyake, A., Friedman, N. P., Emerson, M. J., Witzki, A. H., Howerter, A., 
and Wager, T. (2000). The unity and diversity of executive functions and their contributions to complex "frontal lobe" tasks: A latent variable analysis. Cogn. Psychol., 41, 49-100.

Monsell, S. (2003). Task switching. Trends Cogn. Sci. (Regul. Ed.), 7, 134-140.

Navon, D. (1977). Forest before trees. The precedence of global features in visual perception. Cognitive Psych. 9, 353-383.

Raven, J. C., Court, J. H., and Raven, J. (1988). Manual for Raven's Progressive
Matrices and Vocabulary Scales. London: Lewis.

Trick, L. M., Jaspers-Fayer, F., and Sethi, N. (2005). Multiple-object tracking in children: the "Catch the Spies" task. Cogn. Dev., 20, 373-387.

Waszak, F., Hommel, B., and Allport, A. (2003). Task-switching and long-term priming: role of episodic stimulus-task bindings in task-shift costs. Cogn. Psychol., 46, 361-413.

Conflict of Interest Statement: The authors declare that the research was conducted in the absence of any commercial or financial relationships that could be construed as a potential conflict of interest.

Received: 23 February 2010; paper pending published: 27 February 2010; accepted: 17 March 2010; published online: 21 April 2010.

Citation: Colzato LS, van Leeuwen PJA, van den Wildenberg WPM and Hommel $B$ (2010) DOOM'd to switch: superior cognitive flexibility in players of first per- son shooter games. Front. Psychology 1:8. doi: 10.3389/fpsyg.2010.00008

This article was submitted to Frontiers in Cognition, a specialty of Frontiers in Psychology.

Copyright (c) 2010 Colzato, van Leeuwen, van den Wildenberg and Hommel. This is an open-access article subject to an exclusive license agreement between the authors and the Frontiers Research Foundation, which permits unrestricted use, distribution, and reproduction in any medium, provided the original authors and source are credited. 